\title{
Efficient wide-field VLBI imaging
}

\author{
Olaf Wucknitz ${ }^{* \dagger}$ \\ Argelander-Institut für Astronomie, Auf dem Hügel 71, 53121 Bonn, Germany \\ E-mail: wucknitzeastro.uni-bonn.de
}

Wide-field imaging of the radio sky at milli-arcsec resolution is currently restricted to limited numbers of small fields around known sources, due to the immense computational effort needed to image large fields. With the newly developed "Fast Wide-Field Mapping" algorithm, the computational efficiency is increased by orders of magnitude, making wide-field imaging with VLBI possible routinely. In the future, this method will hopefully help to bring the computational burden in SKA imaging down to a manageable level. This contribution describes the idea and reports on results from first tests.

10th European VLBI Network Symposium and EVN Users Meeting: VLBI and the new generation of radio arrays

September 20-24, 2010

Manchester Uk

* Speaker.

$\dagger$ This work is supported by the Emmy-Noether-Programme of the 'Deutsche Forschungsgemeinschaft', reference WU 588/1-1, and by a Marie Curie European Reintegration Grant within the 7th European Community Framework Programme, Contract No. PERG02-GA-2007-224897 'WIDEMAP'. 


\section{Introduction}

VLBI offers the highest resolution of all astronomical observing techniques. For moderate numbers of pixels, this implies very limited field sizes, often of the order of arcsecs.

The hard field size limit of single pointings is given by the primary beam of the telescopes. Here we discuss the effects of bandwidth smearing and time-averaging smearing, both of which typically become relevant on much smaller scales than the primary beam. In order to overcome these limits, the correlator output must be provided with very high resolution in frequency and time, which causes the data volume to grow roughly linearly with the field area.

In addition, the computing time required to image large fields with standard techiques scales linearly with the field size and with the data volume, which results in a total scaling with (area $)^{2}$ or $(\mathrm{fov})^{4}$. In realistic scenarios, this scaling makes it virtually impossible to image complete primary beams with VLBI resolution. For the long-baseline configurations of the SKA, this problem adds to the numerical effort that is challenging already for small fields.

\section{Primary beam}

The primary beam size for a telescope diameter $D$ at wavelength $\lambda$ is given by $\Theta_{\mathrm{PB}} \approx \lambda / D$, and is independent of the baseline length $L$ and the corresponding resolution $\Delta \theta \approx \lambda / L$. Neglecting factors of order one (oversampling of the resolution elements, shape of the primary beam), we can estimate the maximum number of pixels within the primary beam to be

$$
N_{\mathrm{PB}} \approx\left(\frac{\Theta_{\mathrm{PB}}}{\Delta \theta}\right)^{2}=\left(\frac{L}{D}\right)^{2} .
$$

For a typical global VLBI experiment with $L=10000 \mathrm{~km}$ and $D=25 \mathrm{~m}$, this corresponds to $N_{\mathrm{PB}} \approx$ $1.6 \times 10^{11}$ or about 160 Gigapixels! The exact number would be even higher.

\section{Imaging basics}

The visibility $V$ for relative telescope positions in units of wavelength $(u, v, w)$ in the case of an (incoherent) sky brightness distribution $I(l, m)$ on the unit sphere $\left(l^{2}+m^{2}+n^{2}=1\right)$ is given by

$$
V(v, t)=\iint \frac{\mathrm{d} l \mathrm{~d} m}{\sqrt{1-l^{2}-m^{2}}} I(l, m) \mathrm{e}^{2 \pi \mathrm{i}\left[u l+v m+w\left(\sqrt{1-l^{2}-m^{2}}-1\right)\right]} .
$$

The scaling factor in the denominator can be absorbed into $I(l, m)$ and is not of concern. The additional phase contribution scaling with $w$, the radial component of the baseline, on the other hand, limits the field size if standard techniques are used. For sufficiently small fields $(l, m \ll 1)$, the relation reduces to a two-dimensional Fourier transform:

$$
V(v, t) \approx \iint \mathrm{d} l \mathrm{~d} m I(l, m) \mathrm{e}^{2 \pi \mathrm{i}(u l+v m)}
$$

Inverting the transform by using only the measured visibilities leads to the 'dirty map' which has to be deconvolved with the 'dirty beam' (or PSF) to obtain the final 'clean map'. This poster is about the efficient computation of the dirty map. 
In order to utilise the simple Fourier relationship, the full field of view can be subdivided into small facets for which the approximation still holds. Because the visibilities (and $u v$ coordinates) have to be rotated to new phase centres, the computing time for the dirty image scales with the data volume and with the total field area.

Without the $w$-term, one Fast Fourier Transform (FFT) could be used to create the full field of view at once, taking advantage of the efficiency of the FFT to achieve a much weaker scaling. A clever algorithm coming close to this efficiency even in the general case is the $w$-projection in which the additional phase term is applied by a convolution in $u v$ space (e.g. Cornwell et al., 2008).

However, in addition to the additional convolution (which is combined with the gridding convolution), the application of the FFT requires one huge field for the gridded visibilities. This data field does not fit into the memory of standard computers, so that much less efficient disk-based algorithms have to be applied for the gridding and the Fourier transform. Because of this complication, some faceting is advantageous even in this case.

\section{Smearing}

Because the $u v$ coordinates are measured in units of the wavelength, they vary with frequency. If wide bands are averaged together into single channels, the measured signal is assigned to single $u v$ points even though it really belongs to a significant radial range. This is equivalent to a radial stretching of all sources, called bandwidth smearing, by $\Delta \theta_{\mathrm{BW}}=(\Delta v / v) \theta$. To keep this below the resolution, the field size has to be (much) smaller than $\Theta_{\mathrm{BW}} \approx \Delta \theta(v / \Delta v)$.

A similar effect results from the fact that the $u v$ coordinates are time-dependent and are averaged over finite intervals $\Delta T$, causing time-averaging smearing. The field size is limited to $\Theta_{\mathrm{TA}} \approx \Delta \theta(1$ day $) /(2 \pi \Delta T)$.

In order not to reduce the field of view below the primary beam size, the fractional bandwidth per channel and the integration times have to be limited by

$$
\frac{\Delta v}{v}<\frac{D}{L} \quad, \quad \frac{2 \pi \Delta T}{1 \text { day }}<\frac{D}{L}
$$

These effects cause a scaling of the total data volume with $(L / D)^{2}=N_{\mathrm{PB}}$ which is particularly severe for VLBI.

\section{Practical faceted imaging}

For faceted imaging, the visibilities have to be shifted to the new phase centres before they are gridded. In order to accelerate the gridding process, they can then be averaged in time and frequency corresponding to the much weaker limits for the individual facet. For this strategy, the full-resolution data set has to be processed once for each facet, which causes the total scaling of execution time with $N_{\mathrm{PB}}^{2}$ and makes this practically impossible.

\section{Fast Wide-Field Mapping (FWFM)}

The total computation time is dominated by the shifting and averaging of the data set for many field centres. The imaging of the facets itself scales only much weaker (with $N_{\mathrm{PB}}$ ). 
The process can be made much more efficient with a hierarchical approach similar to the ones used in tree-codes or in the FFT. This method works by taking the full-resolution data set, shifting it to four sub-fields, together covering the original field, and averaging it down by a factor of two in time and frequency for each of them. This produces four smaller data sets that have a sufficient resolution to image the smaller fields, but the total size of the data is (almost) unchanged. In the next step, the same process is performed for each of the four sub-fields to form data sets for 16 sub-sub-fields and so on. This only stops when the field size is small enough to avoid the $w$-effects.

In this way the total data volume has to be shifted and averaged only once for each stage of this process. The number of stages scales with the logarithm of the field size, so that the total execution time grows much weaker than before. For facets of $n$ pixels, the scaling (comparing the standard method and FWFM) can be roughly estimated:

$$
T \sim \frac{N_{\mathrm{PB}}^{2}}{n} \quad \longrightarrow \quad T \propto 2 N_{\mathrm{PB}} \log _{2} \frac{N_{\mathrm{PB}}}{n}
$$

For the extreme $N_{\mathrm{PB}}$ stated above and a typical facet size of $n=10^{6}$, the estimated execution time goes down by a factor of almost 5000 !

\section{An experiment with real data}

For this we continue with the $90 \mathrm{~cm}$ wide-field VLBI experiment described by Lenc et al. (2008), in which only small fields around known sources within the primary beam were imaged. With a standard approach, imaging the full field of the phase-calibrator scans would take about 1 year on a standard desktop PC. The new FWFM method implemented in a very simple way and without optimisation in AIPS and ParselTongue took only 40 hours on the same machine. This corresponds to a speedup by a factor of $\sim 220$. The simplified estimates shown above lead to a factor of $\sim 600$, which provides at least the right order of magnitude. A search of the entire field found exactly the same sources as the ones selected and imaged by Lenc et al. (2008).

\section{Conclusions and outlook}

FWFM is an alternative to known methods for wide-field VLBI imaging which increases the efficiency in mapping full fields by several orders of magnitude. This improvement makes it possible to image entire primary beams on a regular basis with modest computing equipment.

Compared to $w$-projection, the new method is conceptually much simpler. Because it is based on image facets, it allows position-dependent calibration in a natural and simple way. Estimates of the advantage for LOFAR and the SKA will be provided soon. Since the computational demands are a huge challenge for the SKA, even much more modest improvements in efficiency would already be welcome.

\section{References}

Cornwell, T., Golap, K., \& Bhatnagar, S. 2008, IEEE Journal of Selected Topics in Signal Processing, 2, 647, astro-ph/0807.4161

Lenc, E., Garrett, M. A., Wucknitz, O., Anderson, J. M., \& Tingay, S. J. 2008, ApJ, 673, 78 